\title{
A RARE PRESENTATION OF PRIMARY CLAVICLE OSTEOMYELITIS IN CHILDREN: SUBLUXATION OF STERNOCLAVICULAR JOINT AND EXPOSED MEDIAL END OF CLAVICLE
}

Rajni Ranjan ${ }^{1}$, Ajay $^{2}$

\section{HOW TO CITE THIS ARTICLE:}

Rajni Ranjan, Ajay. "A Rare Presentation of Primary Clavicle Osteomyelitis in Children: Subluxation of Sternoclavicular Joint and Exposed Medial End of Clavicle". Journal of Evolution of Medical and Dental Sciences 2014; Vol. 3, Issue 57, October 30; Page: 13047-13049, DOI: 10.14260/jemds/2014/3733

\begin{abstract}
Osteomyelitis of clavicle is an uncommon disease. In children its origin is generally haematogenous and in adults it is more commonly a secondary infection seen in association with predisposing factor. We are reporting a case of clavicle osteomyelitis in an 8 year female child. Patient presented with us with exposed medial $1 / 3$ rd of clavicle with subluxated sternoclavicular joint with abscess around the clavicle. X ray was predominantly sclerotic. Patient was managed by excision of projected medial end of clavicle and I/v antibiotic. Excised fragment sent for histopathological examination confirmed the diagnosis of chronic osteomyelitis. Postoperatively patient achieved normal range of movements of involved upper limb. Primary osteomyelitis of clavicle is very rare disease. The radiographic findings in clavicular osteomyelitis are frequently not diagnostic and histopathological examination of the diseased bone is usually required to confirm the diagnosis. The management includes both medical and surgical strategies. A large part of the clavicle can be excised without significant loss of function.
\end{abstract}

KEYWORDS: Clavicle, Osteomyelitis, Sternoclavicular joint, Excision.

INTRODUCTION: Osteomyelitis of clavicle is an uncommon disease. In children its origin is generally haematogenous and in adults it is more commonly a secondary infection. (1) Despite modern surgical technique and advanced antibiotic therapy, osteomyelitis remains a difficult and challenging problem.

CASE REPORT: An 8 year female child presented to us with exposed medial $1 / 3^{\text {rd }}$ of clavicle with subluxatedsterno-clavicular joint. There was history of swelling around medial half of clavicle 3 month back which was associated with high grade fever. Patient was initially treated by local practitioner by some oral medicines. After which symptoms slightly got better but gradually patient developed ulcer over the clavicle along with pus discharge. Clinical examination revealed an ulcerative lesion $(5 \times 2 \times 2 \mathrm{~cm})$ on medial third of right clavicle with irregular margins. Medial $1 / 3^{\text {rd }}$ of clavicle was protruding out and there was foul smelling discharge.

There was no evidence of anterior cervical or axillary lymphadenopathy. Radiological evaluation of right shoulder and clavicle demonstrated subluxation of sterno-clavicular joint. Medial half of clavicle was broadened and sclerotic. Culture of pus discharge revealed growth of Styphylococcusaureus. Patient was managed by excision of projected medial end of clavicle. Bone along with excised tissue was sent for biopsy. Histopathoplogical report confirmed chronic osteomyelitis. Post operatively patient was kept on intravenous antibiotics. Patient was discharged on $11^{\text {th }}$ post operative day on oral antibiotics. Sinus healed in follow-up period. Patient achieved normal range of movement in right shoulder with no weakness. 
DISCUSSION: Non traumatic lesions of the clavicle are rare. According to Franklin et al, $37.5 \%$ of nontraumatic clavicular lesions are neoplasms, another 37.5\% are infections and remaining $25 \%$ represent developmental anomalies. (2) Medial half of clavicle is preferred site of osteomyelitis. (1) Osteomyelitis of clavicle is a rare condition. Primary osteomyelitis of clavicle usually occurs in children. The infection is diaphyseal and caused by haematogenous spread. Staphylococcus aureus is most common causative organism. However anaerobic and mixed infections are also common.(3)

Osteomyelitis in adults is usually secondary infections. This can occur as complications of head and neck surgery or subclavian vein catheterization.(4) Osteomyelitis is a process of devascularisation of the periosteal and endosteal arterial system of bone secondary to increased intra-osseus pressure, the thrombophlebitis and bone infection itself. If diagnosis and adequate treatment not started early, it results in necrosis of bone. Necrosis of large fragment lead to formation of sequestrum. ${ }^{(4)}$

Osteomyelitis of the clavicle can resemble a neoplastic process on radiograph; hence a biopsy may be necessary to confirm diagnosis.(5) Ewing's sarcoma should always be considered when children present with local pain, swelling, fever \& leukocytosis because of its prevalence in children.(1)

Radiological finding of acute or subacute osteomyelitis include soft tissue swelling, periosteal reaction, cortical irregularity and demineralization. In chronic osteomyelitis there may be thick irregular bone, sclerotic bone interspersed with radiolucencies and elevated periosteum. (4)

Despite modern surgical technique and advanced antimicrobial therapy, osteomyelitis remains difficult and challenging problem. Wide surgical debridement is mainstay of the treatment in the chronic conditions. A large part of the clavicle can be excised without loss of function. $(6,7)$

CONCLUSION: The primary osteomyelitis of clavicle is a rare. It should be differentiated from neoplasms like Ewing's sarcoma specially in children. Surgical excision of involved portion of clavicle along with antibiotic therapy is the mainstay of treatment. This surgical excision of a portion of clavicle does not affect the functional status of shoulder girdle.

\section{REFERENCES:}

1. Eugenio O, Gerscovich, Adam Greenspan. Osteomyelitis of the clavicle: clinical, radiological, and bacteriological findings in ten patients. Skeletal Radiol. (1994) 23: 205-210.

2. Franklin JL, Parker JC, King HA. Nontraumatic clavicle lesions in children. J Pediatr Orthop 7: 575.

3. Garcia S, Combalia A. Segur J M, Llovera A J. Osteomyelitis of the clavicle A Case report. Acta Orthopaedica Belgica, (1999) Vol.65-3.

4. Balakrishnan C, Vashi C, Jackson 0, Hess J. Post-traumatic osteomyelitis of the clavicle: A case report and review of literature. Can J Plast Surg. (2008); 16 (2): 89-91.

5. Aggarwal AN, Dhammi IK, Singh AP, Kumar S, Goyal MK. Tubercular osteomyelitis of the clavicle: a report of four cases. J Orthop Surg (Hong Kong). (2009); 17 (1): 123-6.

6. Dugg P, Shivhare P, Mittal S, Singh H, Tiwari P, Sharma A. Clavicular osteomyelitis: a rare presentation of extra pulmonary tuberculosis. Journal of Surgical Case Reports: (2013), Issue 5.

7. Khan SA, Zahid M, Asif N, Hasan AS. Tuberculosis of the Sternoclavicular. Indian J Chest Dis Allied Sci (2002); 44: 271-3. 


\section{CASE REPORT}

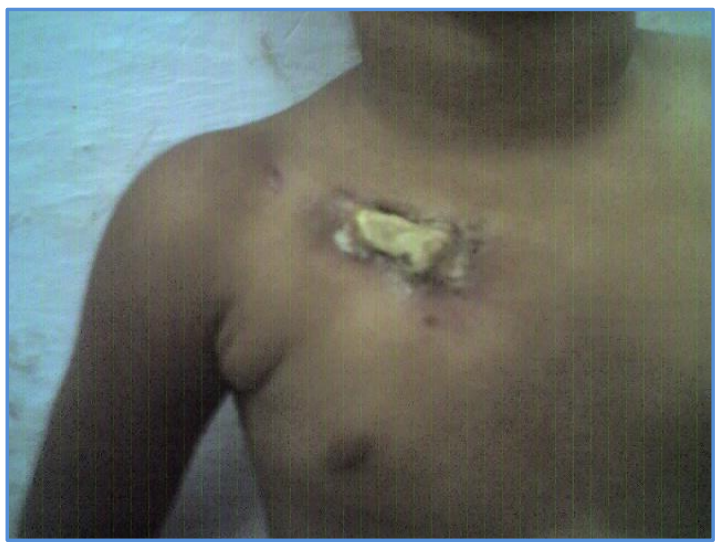

Clinical picture

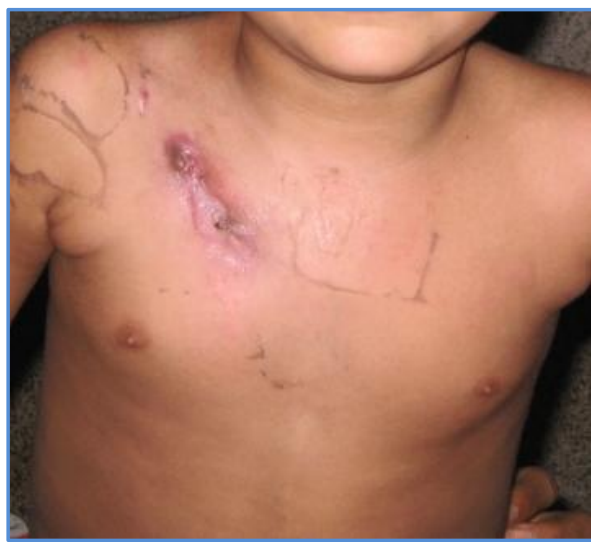

Follow up photograph

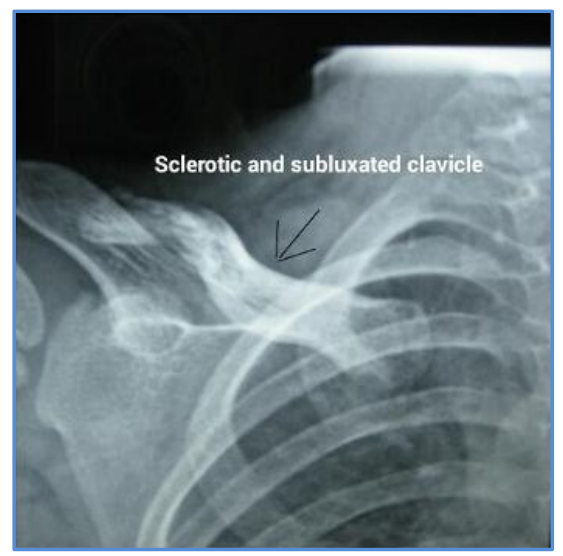

X-RAY

\section{AUTHORS:}

1. Rajni Ranjan

2. Ajay

\section{PARTICULARS OF CONTRIBUTORS:}

1. Assistant Professor, Department of Orthopaedics, School of Medical Sciences \& Research.

2. Assistant Professor, Department of Orthopaedics, School of Medical Sciences \& Research.

\section{NAME ADDRESS EMAIL ID OF THE} CORRESPONDING AUTHOR:

Dr. Rajni Ranjan, \# 802, New Staff Quarter, Sharda Hospital, Knowledge Park-3, Greater Noida, Up-201306.

Email: ranjan974@yahoo.com

Date of Submission: 14/10/2014. Date of Peer Review: 15/10/2014. Date of Acceptance: 28/10/2014. Date of Publishing: 30/10/2014. 